\title{
PAR LA PORTE DÉROBÉE: LA POÉSIE POLONAISE EN FRANÇAIS
}

\author{
Alice-Catherine CARLS (USA)
}

Qui sont les traducteurs et traductrices de poésie polonaise en français? Linguistes, administrateurs de centres culturels, éditeurs, critiques littéraires, historiens de la littérature, diplomates, professeurs de langues ou de litteratures, écrivains, metteurs en scène, poètes, industriels et ingénieurs en retraite, compositeurs de musique, tous ne viennent pas à la langue polonaise de la même façon. Pour les traducteurs de langue maternelle française comme pour ceux de langue maternelle polonaise, les raisons sont multiples: filiation, amour, formation universitaire, lectures, amis, séjours en Pologne ou en France. Toutefois, la connaissance de la langue passe d'abord par le contact avec des êtres humains. C'est ainsi souvent plus par le poète que par le poème que l'on entre en traduction, en y étant convié par un peuple qui croit à sa destinée et à la force historique de sa parole. Qui sont les champions de la poésie polonaise? Après 1945, les efforts d'écrivains et intellectuels émigrés tels Marian Pankowski, Konstanty A. Jeleński, ou Zofia Romanowicz, portèrent leurs fruits; aujourd'hui, tous les traducteurs sont de formidables lobbyistes pour leurs auteurs.

Peut-on, doit-on être traducteur d'une seule langue vers une seule langue? Les premiers traducteurs du polonais vers le français passaient souvent par des langues intermédiaires, telles l'allemand ou le russe. L'influence de l'allemand dans le choix des oeuvres polonaises publiées en France fut et reste toujours visible. Jacques Burko, Roger Legras, et Hélène Włodarczyk sont venus au polonais à travers le russe. Faut-il être poète soi-même pour traduire de la poésie? Maria Delaperrière et Dorota Walczak sont universitaires et historiennes de la littérature et de la civilisation. Maryla Laurent-Zielińska et Laurence Dyèvre sont enseignantes et font un impressionnant travail au niveau des échanges culturels et littéraires entre la France et la Pologne. Christophe (Krzysztof Andrzej) Jeżewski est musicologue et poète, et il traduit aussi bien vers le polonais que vers le français. Peut-on, doit-on ne traduire que de la poésie? Isabelle Macor-Filarska et Erik Veaux traduisent de la prose et des ouvrages historiques, politiques, ou des essais.

Rita Gombrowicz me confiait il y a quelques années qu'elle avait une préférence 
pour les traductions à quatre mains. Pour des raisons linguistiques, tout d'abord - un traducteur polonais pour expliquer, un traducteur français pour peaufiner. Souvent, les traductions se font en tandem; autrefois, le nom du traducteur secondaire (époux ou épouse, ami/e) n'était pas mentionné. Maintenant, il n'est pas rare de voir une traduction signée par deux personnes. La fin de la guerre froide, qui permit de multiplier les contacts entre poètes vivant en Pologne et traducteurs, n'a guère modifié le paysage de la traduction. Aujourd'hui encore, un traducteur de poésie ne peut s'y consacrer qu'à temps partiel. Le traducteur est formé soit à la traduction, soit au polonais, soit à la poésie; encore plus rarement le traducteur est-il formé à la traduction poétique du polonais en français ou la pratique-t-il en exclusivité. Toutefois, on remarque deux tendances nouvelles: d'une part, le nombre croissant de personnes de langue maternelle polonaise qui traduisent de la poésie, et de l'autre la formation linguistique rigoureuse des traducteurs (jusqu'à sept ans de spécialisation) grâce au développement de l'enseignement des langues slaves en France et dans les pays francophones.

Quel suivi accompagne la formation initiale du traducteur? Discussions entre collègues, séminaires, et conférences, bien sûr. Un réseau de recherche et de séminaires organisé par Maryla Laurent-Zielińska et Elżbieta Skibińska autour de leurs universités respectives, l'université Charles-de-Gaulle/Lille III et l'université de Wrocław, réunit ainsi depuis 1995 des traducteurs de l'Europe entière. Partant d'un examen de la traduction littéraire du polonais en français, les publications résultant de ces colloques se sont étendues depuis 2010 à la littérature européenne. Les seize volumes parus à ce jour représentent un imposant travail de réflexion qui va d'oeuvres et d'auteurs particuliers à des analyses plus générales. ${ }^{1}$ Espérons y voir une nouvelle direction qui aidera les traducteurs à sortir de leur isolement.

Si l'on se tourne maintenant vers le monde de l'édition, il y a lieu d'être optimiste. En 2014, la Pologne étant mieux connue des frileux Européens de l'Ouest, et les déplacements culturels étant facilités par l'espace Schengen, le nombre de traducteurs multiculturels et polyglottes a considérablement augmenté. Il en résulte un grand foisonnement de publications de tout format et un nombre croissant de traductions. Quatre jalons importants ont été posés depuis les années 1960. Tout d'abord, l'excelente Anthologie de la poésie polonaise établie par Konstanty A. Jeleński en 1965 et qui fit l'objet d'une réédition en 1981. Jeleński, qui lui-même traduisit plusieurs textes, fit appel à de très grands noms, ${ }^{2}$ ce qui témoigne de sa volonté de confier les traductions à des personnes ayant une relation privilégiée avec la poésie. Puis l'oeuvre de maisons d'édition telles L'Age d'Homme et Noir sur Blanc. ${ }^{3}$ Basées toutes deux en Suisse, elles

\footnotetext{
${ }^{1}$ Le Réseau thématique de recherche et de formation à la recherche. Equipe Cecille, [online - accès juin 2014]: http://cecille.recherche.univ-lille3.fr/axes-de-recherche/lexique-ettraduction/partenaires-56/.

${ }^{2}$ C. Jelenski, Anthologie de la poésie polonaise: 1400-1980, nouvelle édition revue et mise à jour avec la collaboration de Z. Bobowicz, préface de Cz. Miłosz, [Lausanne] 1981. Première édition en 1965, épuisée en 1977. Les traductions sont signées par Yves Bonnefoy, Michel Manoll, Marian Pankowski, Pierre Emmanuel, Allan Kosko, Anne-Marie de Backer, Jean Bourrilly, Charles Dobzynski, Paul Eluard, Marcel Béalu, Arthur Haulot, Jacques Audiberti, André Wat, Paul Cazin, O. V. De L. Milosz, Lucien Feuillade, Michel Boujut, Alain Bosquet, Roger Caillois, et Georges Sidre, pour ne citer qu'eux.

${ }^{3}$ A. Mickiewicz, Ballades, romances, et autres poèmes, traduction de R. Legras, Lausanne 1998; C. K. Norwid, Poèmes, edité par R. Legras, Lausanne 1999; Les poètes polonais du "Scamandre", edité par R. Legras, Lausanne 2004; C. K. Norwid, Vade-mecum, Traduit par Ch. Jezewski et J. Fert, Montricher 2004. On ne peut ici que citer quelques titres et souligner les contributions de leurs traducteurs.
} 
firent beaucoup pour faire connaître la poésie et la littérature polonaises dans les pays francophones. Ensuite, l'anthologie allemande de Karl Dedecius, publiée en français en 2000 intégralement par Nour sur Blanc, et qui contient un choix très sûr des "grands" noms de la poésie polonaise. ${ }^{4}$ Puis des anthologies et études plus pointues telles celles dirigées par Daniel Beauvois ou Maria Delaperrière, qui se spécialisent dans le 20ème siècle. ${ }^{5}$ Il faut néanmoins souligner que, souvent, le lecteur français ne découvre l'oeuvre poétique d'un auteur que des années après que ses romans, essais, ou pièces de théâtre aient été traduits. Cette percée tardive de la poésie est troublante; tel fut le cas pour Czesław Miłosz, Tadeusz Różewicz, Aleksander Wat, et Jarosław Iwaszkiewicz.

Il existe en France, aujourd'hui, deux grandes collections de poésie, Gallimard/ Poésie, dirigée par André Velter, et La Différence/Orphée, dirigée par Claude Michel Cluny. Le nom "Pologne" ne figure pas au catalogue Gallimard/Poésie; par contre O. V. De Milosz y est à l'honneur. ${ }^{6}$ Les grands "classiques" sont à l'honneur chez Orphée Jan Kochanowski, Adam Mickiewicz, Jan Lechoń, Julian Tuwim, et Zbigniew Herbert. Chez d'autres éditeurs se publient maintenant beaucoup les poètes de l'entre-deux guerres. Les poètes de la deuxième guerre mondiale et de l'Insurrection de Varsovie ont attendu assez longtemps, mais Hanna Konicka et Erik Veaux viennent de traduire Anna Świrszczyńska. ${ }^{7}$ Pour l'après 1945, les plus grands noms et les Prix Nobel, tels Czesław Miłosz, Tadeusz Różewicz, Wisława Szymborska, Zbigniew Herbert, Adam Zagajewski, et Miron Białoszewski, sont beaucoup traduits. Les éditions Le Bruit du Temps viennent d'achever la publication de l'oeuvre complète de Zbigniew Herbert en six volumes. Trois des volumes réunissent les oeuvres poétiques complètes, traduites par Brigitte Gautier. ${ }^{8}$ Les poètes de la génération de 1968 tels Ewa Lipska ou Halina Poświatowska commencent à être mieux connus. Les nouveaux catalogues sont prometteurs: outre L'Age d'Homme (Anna Świrszczyńska, 2012) et La Différence / Orphée (Aleksander Wat, 2013; Jarosław Iwaszkiewicz, 2015), les éditions Grèges ont publié un volume de poésies d'Urszula Kozioł en 2012 et préparent un volume de poésies de Krzysztof Siwczyk en 2015, tous deux traduits par Isabelle Macor Filarska. Un volume de Joanna Pollakówna vient d'être publié par Editinter, qui fut le premier à publier Anna Frajlich. ${ }^{9}$ La revue Poésie Première fondée par Robert Dadillon, directeur d'Editinter, publie

\footnotetext{
${ }^{4}$ K. Dedecius, Panorama de la littérature polonaise. Poésie, 2 vol., [Montricher] 2000. Les traducteurs comprennent Claude-Henry du Bord, Christophe Jeżewski, Robert Bourgeois, Jacques Burko, Alice-Catherine Carls, Jacques Dongui et Michel Masłowski, Grażyna Erhard, Dorota Felman et Jacques Jouet, Piotr Kamiński, Roger Legras, Katarzyna Maciejewska, Isabelle Macor-Filarska, Katarzyna Skansberg, et Małgorzata Zakrzewska.

5 J. Donguy, M. Masłowski, Poésie polonaise contemporaine, Paris 1983; Poètes de l'apocalypse. Anthologie de poésie en polonais, hébreu et yiddish (1939-1945). Ouvrage collectif publié sous la direction de D. Beauvois, S. Beres, J.-M. Delmaire, M. Laurent, Lille 1991; M. Delaperrière, La poésie polonaise du vingtième siècle: voix et visages, Paris 2004; R. Legras, Les poètes polonais du "Scamandre", [Lausanne] 2004; Femme de lettres polonaise: Wisława Szymborska, Dorota Masłowska, Catherine Radziwill, Anna Langfus, Anna Szatkowska, Anna Świrszczyńska, Livres Groupe/General Books LLC, 2010.

${ }^{6}$ O. V. De Milosz, La Berline arrêtée dans la nuit; anthologie poétique, [Paris] 1999.

${ }^{7}$ A. Świrszczyńska, Tous les coups sont permis: Poèmes choisis et traduits du polonais, traduction de H. Konicka et E. Veaux, Lausanne 2012.

${ }^{8}$ Z. Herbert, Code de lumière (2011); Monsieur Cogito (2012); et Épilogue de la tempête (2014), traductions de B. Gautier, Paris 2011-2014.

${ }_{9}^{9}$ A. Frajlich, Le Vent, à nouveau me cherche, traduction d'A.-C. Carls, présentation par J. Zieliński, Editinter 2009. Edition spéciale Biennales de la MIPAH, 2012. J. Pollakówna, Avare clarté, traduction d'A.-C. Carls, préface de J. Zieliński, Editinter 2014.
} 
régulièrement des auteurs polonais depuis presque vingt ans et a été la première à faire connaître en France les poèmes de Józef Wittlin, Aleksander Wat, Anna Frajlich, et Tymoteusz Karpowicz, ainsi que celles du poète polono-américain Stuart Dybek.

Depuis dix ans, l'édition a beaucoup changé. On a annoncé la mort des petites maisons d'édition et l'indifférence du public. Ce pessimisme n'est pas justifié. Outre les maisons d'édition qui se sont beaucoup occupées de la Pologne (Denoël, les Editions du Rocher, Libella fondée dans les années 1950 par le regretté Kazimierz Romanowicz, Actes-Sud, L'Harmattan), des maisons d'édition peut-être moins connues mais non moins sérieuses ont pris le relai (Grèges, Le Bruit du Temps). Il est certain qu'il y a un écart de génération entre les éditeurs de facture traditionnelle, et les jeunes éditeurs favorables aux multimedia qui leur permettent de contourner les exigences commerciales et publicitaires. Les revues publiant de la poésie polonaise en traduction ne manquent pas. Elles sont trop nombreuses pour être nommées toutes ici, mais on peut en citer deux: Europe est la doyenne des revues ouvertes à l' "autre Europe," et reste fidèle à l'impression sur papier. Recours au poème est une jeune revue publiée en ligne. Dirigée par Matthieu Baumier et Gwen Garnier-Duguy, elle s'est assurée de la collaboration régulière d'Andrzej Taczyński, un jeune poète né en Pologne et résidant aux Etats Unis et en France, et de celle d'Isabelle Macor Filarska. ${ }^{10}$ Les biennales et festivals de poésie qui ont lieu en France et dans les pays francophones font aussi une belle part à la poésie polonaise. Il ne faut pas manquer ici de rappeler les contributions des pays voisins de la France, notamment celles de Fallois / L'Âge d'Homme en Suisse, et de Noir sur Blanc, fondé en Suisse et devenu maintenant le groupe international Libella. La Maison Internationale de Poésie Arthur Haulot en Belgique a dès sa fondation ouvert ses portes aux poètes polonais, à commencer par Aleksander Wat qui assista à la quatrième Biennale de Poésie à Knokke-le-Zoute en 1959. La revue de la MIPAH, Le Journal des Poètes, a toujours généreusement accueilli les poètes polonais et continue désormais sous l'égide de la maison d'édition Taillis du Pré.

L'importance de la diffusion sur le web est soulignée par le site du fonds littéraire Jacques Burko inauguré en 2010. Ses traductions de poèmes par Wisława Szymborska et Jerzy Ficowski y figurent en pdf avec le texte des communications inaugurales. Comme le signale Berthe Burko-Falcman, Jacques Burko, poète et traducteur de poèmes russes en tant qu'étudiant, devint un passeur passionné de poésie polonaise après une longue carrière d'ingénieur.

Dès 1957, il a tenté de faire publier des traductions de Julian Tuwim. En vain. Il y est parvenu en 1993 dans la collection Orphée de Claude-Michel Cluny... Il a pu faire publier des traductions des poètes polonais Zbigniew Herbert, Niemec, Podsiadło, Tomasz Rozycki. D'excellent poètes n'ont pas trouvé d'éditeurs, la poésie se vend mal, je pense en particulier a Edward Stachura, un poète polonais né en France. ${ }^{11}$

Jacques Burko fut, de 2005 à sa mort, directeur de la collection Poésie aux Editions Buchet-Chastel et y publia seize titres. Il a donc laissé une oeuvre impressionnante. Les circonstances jouent souvent de mauvais tours. J'ai ainsi proposé deux volumes pour la collection Orphée: en 1988, un volume Szymborska et, au milieu des années 1990, un

10 "Europe. Revue littéraire mensuelle", [on-line - accès juin 2014]: http://www.europerevue.net/; Maison Internationale de Poésie Arthur Haulot (MIPAH), [on-line - accès juin 2014]: http://www.mipah.be/fr; "Recours au poème. Poésies \& Mondes poétiques", [on-line - accès juin 2014]: http://www.recoursaupoeme.fr/.

${ }^{11}$ Inauguration du fonds littéraire „Jacques Burko”. 10 décembre 2010, [on-line - accès juin 2014]: http://www.pulrulczyk.net/mapage6/compte-rendu-manifestation-du-10-d-cembre-site-2-.pdf. 
volume Wat. La collection Orphée cessa de paraître de 1998 à 2012, et le volume Wat ne vit le jour qu'en 2013. L'attribution du Prix Nobel à Wisława Szymborska en 1996 fut suivi d'un contrat de traduction exclusif attribué à Piotr Kamiński. ${ }^{12}$ Les autres traducteurs potentiels de Szymborska - et ils sont nombreux — commencent tout juste timidement à publier quelques traductions sur le web.

Le web est un véhicule important pour briser la timidité vis-à-vis de poètes moins connus. Plusieurs sites web auto-publiés portent sur des thèmes pointus et utilisent le logiciel "WordPress" en assurant les copyrights du traduceur par le programme "Creative Commons." "Pologne immortelle, par Hermine", ${ }^{13}$ présente des traductions de Jan Kochanowski, Julian-Ursyn Niemcewicz, Adam Mickiewicz, Cyprian-Kamil Norwid, Karol Wojtyła (plus connu comme le pape Jean-Paul II), et Czesław Miłosz. Un autre site, "Poètes polonais", ${ }^{14}$ créé en 2012, publie , avec une brève présentation de chaque auteur, des traductions de poèmes de Romuald Minkiewicz, Władysław Orkan, Marya Szpyrkówna, et Stanisław Korab-Brzozowski. Un blog du Nouvel Observateur publie Czesław Miłosz et Miron Białoszewski à côté de poèmes sans attribution d'auteur ni de traducteur. ${ }^{15}$ Un autre blog un peu plus ancien offre plusieurs poèmes en bilingue, de Adam Mickiewicz, Tytus Czyżewski, Krzysztof Kamil Baczyński, Jan Brzechwa, et Wisława Szymborska. Les textes et traductions sont empruntés aux sites des poètes et renvoient aux liens de provenance avec cette annonce: "Si vous trouvez quelque chose en français... il n'est pas interdit d'en faire profiter la communauté."16 Les sites web libres et les blogs ont l'avantage de faire connaître des textes moins lus et d'échapper aux contraintes éditoriales et financières traditionnelles, mais ils sont pour la plupart limités à des auteurs tombés dans le domaine public. La qualité et la rigueur souffrent quand les textes polonais perdent leurs signes diacritiques ou ne sont tout simplement pas publiés, ou bien quand le nom du traducteur manque. Que deviennent, dans tout cela, les droits d'auteur et de traducteur? Et enfin, quel effet ces partages libres ont-ils sur les professionnels de la traduction et de l'édition?

Parlons, enfin, de la traduction. Les nouveaux traités sur l'art de la traduction présentent les traducteurs comme des passeurs de culture et de contexte (subtexte, paratexte). Les théories linguistiques postmodernes nous ont rendus conscients non seulement de la langue et de la culture qui entourent un poète, mais de l'usage qu'il en fait, et de l'importance de sa personnalité. La traduction doit être traitée comme l'alpha et l'omega du travail, et le poème doit être examiné à des niveaux multiples. On ne saurait traduire Aleksander Wat, par exemple, sans connaître non seulement le contexte historique objectif des événenments qui marquèrent sa vie, les cultures polonaise et

${ }^{12}$ A. Wat, Les quatre murs de ma souffrance, traduit par A.-C. Carls, présentation par J. Zieliński, Paris 2013; W. Szymborska, De la mort sans exagérer, traduit par P. Kamiński, Paris 1996. Laurent Bayart dont la maison d'édition L'Ancrier venait de publier un volume Szymborska, dut le mettre au pilon. Laurent Bayart anime désormais un site web; http://www.laurent-bayart.fr.

${ }^{13}$ La Poésie polonaise, 7 grands poètes (publié le 06.05.2012), Pologne Immortelle, [on-line - accès juin 2014]: http://pologneimmortelle.wordpress.com/.

${ }^{14}$ Poètes polonais. Découvrez les poètes polonais de la Jeune Pologne (20ème siècle), présentation par Chantal Lainé, 11 janvier 2014, [on-line - accès juin 2014]: http://poetespolonais. wordpress.com.

${ }^{15}$ Poésie polonaise. Le Nouvel Observateur (25.08.2012), [on-line - accès juin 2014]: http://poesiepolonaise.blogs.nouvelobs.com.

16 Laurent, Poésie polonaise (27.10.2007), [on-line - accès juin 2014]: $\mathrm{http}: / /$ lespolonais.forumpro.fr/t50-poesie-polonaise. 
juive de son époque, et les relations entre Juifs et Polonais, mais aussi son immense culture personnelle et ses lectures, parmi lesquelles la Bible tenait une grande place. Il ne faut pas non plus oublier ses traditions familiales ni sa personnalité qui sont évoquées dans les mémoires de son épouse Ola Wat et dans ses conversations avec Czesław Miłosz. ${ }^{17}$ S'il faut un village pour élever un enfant, il en faut un pour faire une bonne traduction. Plus les références culturelles de l'oeuvre poétique sont discrètes, plus la recherche s'impose. Ainsi en est-il de la poésie d'Anna Frajlich, en apparence facile à traduire; les résonnances contenues dans chaque vers exigent une connaissance approfondie de la littérature polonaise, comme vient de le montrer Iwona Smolka dans son excellente recension du dernier volume poétique d'Anna Frajlich. ${ }^{18}$

Enfin, parlons du problème de la théorie et de la technique. Dans notre ère postmoderne, l'art de la traduction n'est heureusement plus soumis à aucun diktat intellectuel, encore que les traités et autres études se multiplient et que les exigences concernant la qualité et l'étendue des connaissances linquistiques soient inchangées. Les règles à respecter? Celles auxquelles on pense peut-être le moins. Le traducteur doit servir le texte, pas s'approprier un auteur - au sens large du terme. Il faut éviter les traductions palimpsestes. À la langue et la culture françaises (générale et poétique) s'ajoutent l'usage que le traducteur en fait, la langue et la culture polonaise contemporaines du poète, le sens qu'il leur donne, l'usage qu'il en fait, et pour finir l' interprétation de tous ces langages par les conseillers en traduction, dictionnaires et spécialistes. Cela donne une dizaine de voix qui risquent d'alourdir la traduction et de rapetisser le poème original. Comment obtenir une traduction aussi fidèle et aussi transparente que possible? Après une écoute profonde, le traducteur doit s'effacer. Se dépouiller de lui-même. Reconnaître ses limites. C'est au niveau du comportement (lui qui régit l'appareil scientifique, linguistique, technique, érudit, perceptif, et affectif du traducteur) que se décide une bonne traduction.

Est-il facile de respecter l'altérité du texte? Un ouvrage de la collection dirigée par Maryla Laurent-Zielińska semble répondre à cette question. ${ }^{19}$ Traduire, c'est montrer l'Autre par le Verbe. N'est-ce pas là une démarche profondément humaine qui se fonde sur la confiance et le respect? Epouser la pensée d'un autre, comprendre les résonnances de son langage, et les interpréter sans les déformer: dans une bonne traduction, le traducteur est invisible, tout comme dans un bon sermon le pasteur, rabbin, ou prêtre, s'efface pour mieux montrer Dieu. La traduction de la poésie est plus qu'une profession ou un bagage de connaissances. On vit en elle, tout comme on vit en poésie.

\section{THROUGH SECRET DOOR: POLISH POEMS TRANSLATED INTO FRENCH}

Examining the publications and translations of Polish poetry into French since the mid-1960s brings up characteristics that are unique. Polish poetry has been translated into French often and well not only by French publishing houses, but also by Swiss and Belgian publishing houses. There has been a steady number of high-profile publishing houses and magazines interested in Polish poetry. And the translators are a diverse group of individuals with various interests and specializations, who often pursue translation for the love of the work and lobby to get authors

${ }^{17}$ O. Wat, L'ombre seconde, Paris-Lausanne 1989; A. Wat, Mon siècle. Confession d'un intellectuel européen, préface de Cz. Miłosz Paris-Lausanne 1989.

${ }^{18}$ I. Smolka, Pamięć podwojona, Nowe Książki $2013 \mathrm{nr} 9$.

${ }^{19}$ L'autre tel qu'on le traduit, textes réunis par M. Laurent et L. Waleryszak, Paris 2006. 
publicized and published. In the $21^{\text {st }}$ century, poetry is more widely translated; translators receive more systematic linguistic and literary training; and the advent of online poetry magazines and the world of e-books is redefining the way in which the translations are distributed, allowing instant world-wide access.

Keywords: translations into French; Polish poetry. 